Original paper

\title{
Technical challenges for FLASH proton therapy
}

\author{
Simon Jolly ${ }^{\mathrm{a}, *}$, Hywel Owen ${ }^{\mathrm{b}, \mathrm{c}}$, Marco Schippers ${ }^{\mathrm{d}}$, Carsten Welsch ${ }^{\mathrm{b}, \mathrm{e}}$ \\ ${ }^{a}$ University College London, United Kingdom \\ ${ }^{\mathrm{b}}$ Cockcroft Institute of Accelerator Science and Technology, United Kingdom \\ ${ }^{\mathrm{c}}$ University of Manchester, United Kingdom \\ ${ }^{\mathrm{d}}$ Paul Scherrer Institute, Switzerland \\ ${ }^{\mathrm{e}}$ University of Liverpool, United Kingdom
}

\section{A R T I C L E I N F O}

\section{Keywords:}

FLASH

Proton therapy

PBT

Proton FLASH therapy

Proton radiotherapy

Ultra-rapid irradiation

Accelerator

Cyclotron

Synchrotron

Synchro-cyclotron

Linac

Medical accelerator

\begin{abstract}
A B S T R A C T
There is growing interest in the radiotherapy community in the application of FLASH radiotherapy, wherein the dose is delivered to the entire treatment volume in less than a second. Early pre-clinical evidence suggests that these extremely high dose rates provide significant sparing of healthy tissue compared to conventional radiotherapy without reducing the damage to cancerous cells. This interest has been reflected in the proton therapy community, with early tests indicating that the FLASH effect is also present with high dose rate proton irradiation.

In order to deliver clinically relevant doses at FLASH dose rates significant technical hurdles must be overcome in the accelerator technology before FLASH proton therapy can be realised. Of these challenges, increasing the average current from the present clinical range of 1-10 nA to in excess of $100 \mathrm{nA}$ is at least feasible with existing technology, while the necessity for rapid energy adjustment on the order of a few milliseconds is much more challenging, particularly for synchrotron-based systems. However, the greatest challenge is to implement full pencil beam scanning, where scanning speeds 2 orders of magnitude faster than the existing state-of-the-art will be necessary, along with similar improvements in the speed and accuracy of associated dosimetry. Hybrid systems utilising 3D-printed patient specific range modulators present the most likely route to clinical delivery. However, to correctly adapt and develop existing technology to meet the challenges of FLASH, more pre-clinical studies are needed to properly establish the beam parameters that are necessary to produce the FLASH effect.
\end{abstract}

\section{Introduction}

The challenge in radiotherapy has always been to optimise the Tumour Control Probability (TCP) over the Normal Tissue Complication Probability (NTCP) [1]. The introduction of proton beam therapy allowed a similar conformal dose to be delivered to the treatment volume but with a lower dose to the surrounding healthy tissue that improves the TCP/NTCP ratio [2]. Recently, a new method of dose delivery has shown extremely promising results in the sparing of normal tissue with little reduction in tumour control [3]. Termed FLASH radiotherapy, this technique involves delivering the same treatment dose but in much shorter time intervals - fractions of a second as opposed to minutes and in far fewer fractions (or even a single fraction) and therefore at dose rates that are thousands of times higher [4]. First reported in 2014 [5] and confirmed in multiple subsequent experiments (e.g. [6-10]), in several tissue types FLASH has demonstrated that these high dose rates do provide a significant improvement in the TCP/NTCP ratio. A recent first patient treatment indicates this technique has promise [11]. While these experiments were carried out with electrons and X-rays, the similar RBE of protons has also increased interest within the proton therapy community [12] and experiments have shown that such effects could also be achieved using protons [13]. Coupled with the improved dose distribution offered by Proton Beam Therapy (PBT) treatment, the suggestion is that further improvements can be realised when delivering FLASH treatments with protons [14]. The issue, however, with present-day clinical PBT systems is that many of the machine characteristics fall short of the requirements needed for FLASH dose delivery: a summary is given in Table 1 . While experimental setups for delivering FLASH dose rates with existing clinical systems have been realised [15-17], these have so far been limited to small volumes. This is not unexpected since the requirements for conventional PBT dose rate are several orders of magnitude below that required for FLASH.

\footnotetext{
* Corresponding author.

E-mail address: s.jolly@ucl.ac.uk (S. Jolly).
} 
Table 1

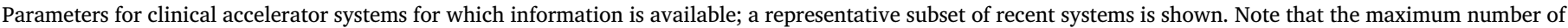

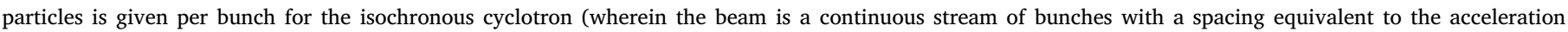

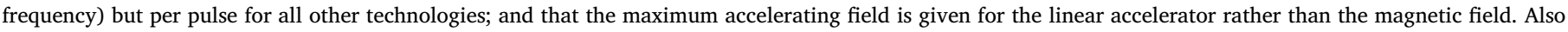

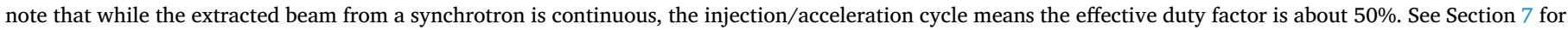
more details.

\begin{tabular}{|c|c|c|c|c|c|c|}
\hline \multirow{3}{*}{$\begin{array}{c}\text { Accelerator Type } \\
\text { Vendor } \\
\text { System }\end{array}$} & \multicolumn{2}{|c|}{ Isochronous Cyclotron } & \multicolumn{2}{|c|}{ Synchrocyclotron } & \multirow{2}{*}{$\begin{array}{c}\text { Synchrotron } \\
\text { Hitachi }\end{array}$} & \multirow{2}{*}{$\begin{array}{c}\text { Linear Accelerator } \\
\text { AVO }\end{array}$} \\
\hline & IBA & Varian & IBA & Mevion & & \\
\hline & $\mathrm{C} 230$ & PROBEAM & $\mathrm{S} 2 \mathrm{C} 2$ & S250 & ProBeam & LIGHT \\
\hline Maximum Energy (MeV) & 230 & 250 & 250 & 250 & 250 & 250 \\
\hline Minimum Energy (MeV) & 70 & 70 & 70 & 70 & 70 & 37.5 \\
\hline Peak Current $(\mu \mathrm{A})$ & 0.3 & 0.8 & $\sim 18$ & $\sim 7$ & $4.8 \times 10^{-3}$ & $\sim 40$ \\
\hline Max Ave. Current (nA) & 300 & 800 & $\sim 130$ & $\sim 32$ & 4.8 & 32 \\
\hline Accel. Frequency (MHz) & 106.1 & 72.8 & $87.6-63.2$ & $133-90$ & $1.3-10$ & 3,000 \\
\hline Repetition rate & CW & CW & $1 \mathrm{kHz}$ & $500-750 \mathrm{~Hz}$ & CW & $200 \mathrm{~Hz}$ \\
\hline Treatment Pulse Length & $>400 \mu \mathrm{s}$ & $>400 \mu \mathrm{s}$ & $7 \mu \mathrm{s}$ & $6 \mu \mathrm{s}$ & $0.5-5 \mathrm{~s}$ & $4 \mu \mathrm{s}$ \\
\hline Bunch Length & $\sim 2 \mathrm{~ns}$ & $\sim 2 \mathrm{~ns}$ & $\sim 2 \mathrm{~ns}$ & $\sim 2 \mathrm{~ns}$ & $\sim 25-200 \mathrm{~ns}$ & $\sim 0.5 \mathrm{~ns}$ \\
\hline Max Part. per Bunch/Pulse & 100,000 & 70,000 & $8 \times 10^{8}$ & $4 \times 10^{8}$ & $1.5 \times 10^{11}$ & $10^{10}$ \\
\hline Electric/Central Field & $1.7 \mathrm{~T}$ & $2.4 \mathrm{~T}$ & $5.75 \mathrm{~T}$ & $9 \mathrm{~T}$ & $1.7 \mathrm{~T}$ & $25 \mathrm{MV} / \mathrm{m}$ \\
\hline References & {$[18,19]$} & {$[20,21]$} & {$[22]$} & [23-25] & {$[26-28]$} & {$[29,30]$} \\
\hline
\end{tabular}

\section{Clinical dose rate requirements}

A common clinical specification for minimum dose rate for conventional proton therapy is to be able to deliver a dose of 2 Gray for a single field to a 1 litre volume in 1 minute [31,32]. For a cubic $10 \times 10 \times 10 \mathrm{~cm}^{3}$ volume that lies from $10-20 \mathrm{~cm}$ deep within another larger water volume, this corresponds to $1.9 \times 10^{11}$ protons. ${ }^{1}$ This value is important for future discussions since, by scaling up the dose rate from conventional to FLASH PBT and assuming the same fractionation scheme, one can set a lower limit on the total number of protons and the proton rate needed for FLASH delivery. $1.9 \times 10^{11}$ protons is equivalent to $\sim 30 \mathrm{nC}$ : for dose delivery at FLASH rates, wherein all the dose is delivered in $\sim 100 \mathrm{~ms}$, this corresponds to a beam current of $300 \mathrm{nA}$ at the patient, or a dose rate of $20 \mathrm{~Gy} / \mathrm{s}$. As such, the accelerator and beam delivery system must be capable of delivering an average current of $300 \mathrm{nA}$ to the patient in order to meet the current clinical specification: of the existing medical proton accelerator technologies, only isochronous cyclotrons are capable of delivering such a sustained current and then only at the highest energies (see Table 1): losses in the beam transport between accelerator and patient also reduce the intensity at the patient, particularly at low energies. A volume perhaps closer to standard clinical scenarios is one of $5 \times 5 \times 5 \mathrm{~cm}^{3}$ ie. a $125 \mathrm{ml}$ volume that is an eighth of the standard 1 litre clinical volume: if one considers such a volume lying $15-20 \mathrm{~cm}$ deep within another larger water volume, this requires $2.8 \times 10^{10}$ protons and therefore a charge of $\sim 4 \mathrm{nC}$ or a current of $40 \mathrm{nA}$ for delivery within $100 \mathrm{~ms}$.

A consensus on the beam parameters that can be considered as FLASH has not yet been established: the exact specification is likely to be an interdependent combination of dose, dose rate, repetition rate and number of pulses [35]. The effects of multiple fields is also not yet known, and there remain many open questions as to the cumulative effect of FLASH doses delivered either to different regions of one tumour volume, or from different field directions to the same volume. However, the commonly accepted definition is for dose rates above $40 \mathrm{~Gy} / \mathrm{s}[35,36]$. Assuming that $40 \mathrm{~Gy} / \mathrm{s}$ dose rates can be delivered for $100 \mathrm{~ms}$, this equates to a total dose of $4 \mathrm{~Gy}$ or $3.8 \times 10^{11}$ protons for the standard 1 litre clinical volume quoted above, equivalent to a $\sim 60 \mathrm{nC}$ of charge or $600 \mathrm{nA}$ average current, or an average current of $40 \mathrm{nA}$ for the smaller $125 \mathrm{ml}$ subvolume. This is the specification for delivered beam current used in subsequent calculations. The particular values for some example treatment plans, including the ones described above, are given later in Table 2. This assumes that the dose averaged dose rate

\footnotetext{
${ }^{1}$ Treatment planning calculation carried out by Christian Gräff, GSI, using the TRiP98 treatment planning software $[33,34]$.
}

(DADR) is the figure of merit [14], wherein the entire dose must be delivered at a rate $\geq 40 \mathrm{~Gy} / \mathrm{s}$ and within $100 \mathrm{~ms}$ for the entire volume, utilising a single field. It may be that FLASH effects can also be achieved by delivering at such rates to smaller sub-volumes within a larger treatment volume: this would thereby relax the time constraint and allow high doses to be delivered in short pulses. It is plausible that the figures of merit might be the delivery time and dose rate not to the tumour but to healthy tissue: as such, treatment scenarios - particularly with multiple fields - may eventually be realised wherein the dose delivery to the entire tumour volume falls below the minimum FLASH requirements but the healthy tissue-sparing FLASH effect can still be realised. However, for the purposes of the subsequent discussion and calculations, a DADR of $40 \mathrm{~Gy} / \mathrm{s}$ is assumed for a single field plan, thereby requiring an average current of $600 \mathrm{nA}$ for the 1 litre volume or $40 \mathrm{nA}$ for the smaller subvolume; this is the minimum requirement for delivering FLASH proton therapy considered here.

Even for the larger volume, such a dose rate is not very far from the currents available using some existing PBT accelerator technologies $[37,38]$ - for example, the PSI COMET cyclotron is capable of delivering a sustained extracted current of $800 \mathrm{nA}[20,39]$ - but certain improvements would be needed to ensure a clinically safe system, not least of which is ensuring safe delivery of a specified dose to a treatment volume (see Section 4.2). Whilst modern isochronous cyclotrons are capable of delivering large currents, one important limitation they have is that lossy energy degraders must be used to select treatment energies lower than the fixed extraction energy of the cyclotron. Beam losses due to the degrader at the lowest delivered energies around $70 \mathrm{MeV}$ can exceed $99 \%$, which precludes the use of certain beam delivery modalities for FLASH unless increased beam currents are available: the various beam delivery options are discussed in Sections 3-5.

The above discussion assumes that one is still mimicking the fractionation regime of current clinical treatment with a single field, whilst treating at FLASH dose rates: in a normal fractionated treatment the total dose (say, $\sim 60 \mathrm{~Gy}$ ) is spread over 30 or so fractions of $2 \mathrm{~Gy}$ each, with multiple fields within each fraction. If hypofractionation is used (wherein the total dose is delivered in only a few fractions or even in a single fraction), that implies a dose rate of $600 \mathrm{~Gy} / \mathrm{s}$. This sets a much more challenging accelerator requirement of $6 \times 10^{12}$ protons or $\sim 1 \mu \mathrm{C}$ of charge for the 1 litre volume. Delivering this in $100 \mathrm{~ms}$ requires an average current of $\sim 10 \mu \mathrm{A}$, well above the capabilities of any existing clinical proton accelerator.

The discussion that follows assumes the minimum FLASH dose rate quoted in the literature of $40 \mathrm{~Gy} / \mathrm{s}$ and therefore a total of $3.8 \times 10^{11}$ protons or $\sim 60 \mathrm{nC}$ of charge at an average current of $600 \mathrm{nA}$, delivered in $100 \mathrm{~ms}$ to a 1 litre volume: where appropriate, comparisons 
Table 2

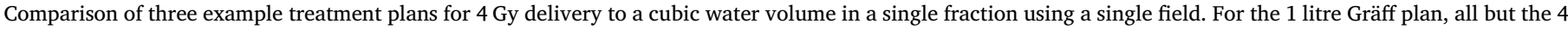
proximal layers have more than 2,500 spots per layer.

\begin{tabular}{|c|c|c|c|c|c|c|c|c|}
\hline \multirow[t]{2}{*}{ Plan Name } & \multirow[t]{2}{*}{ Dimensions } & \multirow[t]{2}{*}{ Total Protons } & \multirow[t]{2}{*}{ Layers } & \multirow[t]{2}{*}{ Total Spots } & \multicolumn{3}{|c|}{ Spots/Layer } & \multirow[t]{2}{*}{ Spot Spacing } \\
\hline & & & & & Min & Max & Ave & \\
\hline Gräff 1 litre & $10 \times 10 \times 10 \mathrm{~cm}^{3}$ & $3.8 \times 10^{11}$ & 34 & 96,222 & 595 & 3,425 & 2,830 & $2 \mathrm{~mm}$ \\
\hline Gräff $125 \mathrm{ml}$ & $5 \times 5 \times 5 \mathrm{~cm}^{3}$ & $5.5 \times 10^{10}$ & 17 & 10,622 & 622 & 625 & 625 & $2 \mathrm{~mm}$ \\
\hline van de Water & $10 \times 10 \times 10 \mathrm{~cm}^{3}$ & $3.3 \times 10^{11}$ & 16 & 1,912 & 7 & 310 & 120 & $1-102 \mathrm{~mm}$ \\
\hline
\end{tabular}

are also given to the smaller $125 \mathrm{ml}$ subvolume. In order to deliver hypofractionated treatment rates any of the quoted times need to be reduced by a factor of 15 . In addition, to simplify the discussion, only single field delivery is assumed.

\section{FLASH proton delivery: double-scattering systems}

The simplest way to meet the current requirement discussed above would be with the more traditional double-scattering arrangement: experimental versions of such systems have already been realised [17]. In utilising patient-specific range shifters and collimators and delivering a constant current, the "active" part of the beamline is reduced only to the range modulator wheel or ridge filter that is used to produce the necessary energy/range variation for the given treatment volume. In comparison to existing clinical PBT systems, a double-scattering FLASH beamline must meet the following additional requirements:

1. The accelerator must be able to deliver a suitably high continuous current such that FLASH dose rates can still be achieved despite the transmission losses of the double scattering system, which can be more than $50 \%$.

2. The accelerator beamline must be able to withstand this higher peak current. In particular, if intercepting diagnostics are permanently inserted into the beamline, the thousandfold increase in instantaneous current must be tolerable.

3. Extra beam losses at collimators, beam diagnostics and rangeshifting materials at the patient will create activation and neutrons: these must be monitored and minimised.

4. The dose-monitoring and beam-interrupting systems must be able to respond quickly enough to shut off the delivered beam once the required dose has been delivered - or in case an error is detected to ensure that the patient is not overdosed.

5. The mechanical system used to produce the variation in range such as a range modulator wheel - must adjust quickly enough that many passes over the treatment volume are possible within the FLASH delivery time to ensure the volumetric dose deposition is not susceptible to temporal changes in the proton rate.

6. The on-line data analysis of the dose monitoring must remain synchronous to the modulated beam energy, in order to obtain the proper dose readout.

Transmission losses through a double-scattering system are usually in the range 50-80\%; with this reduction, the existing PSI COMET cyclotron would be able to deliver a continuous current anywhere from $160-400 \mathrm{nA}$ at the patient, capable of meeting the maximum current requirement for the $125 \mathrm{ml}$ volume but falling short of the clinical requirements for the 1 litre volume set out earlier in Section 2. As such, more efficient scattering systems in combination with a larger proton source current will be required to meet the $40 \mathrm{~Gy} / \mathrm{s}$ FLASH rate with a double-scattering system: improvements in transmission are, however, likely to be modest.

Intercepting diagnostics do exist that can withstand much higher currents than those that will be encountered in FLASH treatments: in addition, it is not an absolute necessity to utilise permanently- intercepting diagnostics, given the large range of non-intercepting diagnostics already in use in modern accelerators [40]. Together with Points 1 and 5 above, this weighs against the use of accelerator technologies that give pulsed beam delivery output, such as synchro-cyclotrons and linacs (see Sections 8 and 9). In particular, as with existing facilities a FLASH double-scattered system clearly favours the use of isochronous cyclotrons: a bunch acceleration frequency in excess of $10 \mathrm{MHz}$ gives an effectively continuous beam, and along with the stability of the extracted current ensures no interplay between any range modulator and the beam bunch structure. Pulsed beams may be possible if the time structures in which beam delivery systems are working do not interfere with the time structure of the beam pulses, for example by using a ridge filter instead of a modulation wheel. Synchrotrons are less attractive because of the increase in cost and complexity needed to meet the higher dose rate specification (see Section 7).

Being able to actively monitor the delivered dose is probably the most challenging area in realising such a clinical system. The dose monitor must be able to accurately measure peak dose rates thousands of times higher than existing clinical systems and react with equal speed in order to shut off the beam once the correct dose has been delivered. In addition, this dose monitor will need to be able to communicate with the remainder of the accelerator control system with sufficient speed to shut off the beam rapidly enough to prevent significant unwanted dose being delivered. Further discussions on FLASH dosimetry requirements are given in Section 4.2.

Whilst passive scattering offers some advantages over beam scanning for high dose-rate delivery, the limitations of a passive-scattering system are still present:

1. Collimators and range shifters have to be manufactured for each patient and for each field. A multi-leaf collimator (MLC) would provide an alternative, but no clinical systems currently exist. Range shifters or degraders could be replaced by ridge or pin filters, provided the following beam transport system has a sufficiently large energy acceptance: more detail is given in Section 5.2.

2. The dose is not as conformal to the treatment volume since the proximal edge of the dose delivery volume is a shallower replica of the distal edge, and not of the proximal edge of the treatment volume (the depth range is approximately constant across the treated volume).

3. There is a significant increase in neutron dose to the patient as a result of the extra material that intercepts the beam.

It is for these reasons that newly-installed PBT systems predominantly offer spot scanning.

\section{FLASH proton delivery: spot scanning}

Whilst mitigating the shortcomings of passively scattered beamlines outlined above, delivering FLASH PBT with modern spot-scanning systems is considerably more challenging. While the average beam current requirement given above for double-scattered systems still holds, for spot-scanning systems the actual delivered current will be significantly higher since one must account for the dead time between 
spots, for switching energy layers, and for the variation of beam current required in each layer/spot to create a uniform dose distribution. In addition, rather than having a "static" magnetic beamline that does not have to adjust whilst the protons are delivered to the patient, with spotscanning the beamline is "active": steering magnets within the nozzle must direct the pencil beam to the desired spot within the treatment volume and every magnet within the entire beamline must also adjust to match the beam energy for a given energy layer.

In the future, rapid energy variation will demand beam delivery systems with large energy acceptance; rather than adjust the magnet settings for each energy layer, the beam dispersion is limited by a suitable beam-optical arrangement and sufficient aperture within the magnets and vacuum system provided to obtain a large energy acceptance. There are broadly two types of design: smaller-acceptance designs that have an energy acceptance sufficient for a single treatment depth range, in which the gantry optics are often composed of several conventional magnetic achromats [41-43]; and large-acceptance designs which aim to allow any energy from an accelerator source (say, from $70 \mathrm{MeV}$ to $250 \mathrm{MeV}$ ) with no magnetic adjustment at all. For the latter case, FFAG (fixed-field, alternating gradient) optics, or achromatic multipole-based bending systems, are often proposed and since the magnets are fixed one may use more compact designs that utilise either permanent magnets or superconducting magnets to limit complexity and mass [44-47].

In smaller-acceptance systems there is the option of having a rangeshifting device just before the patient, since then the magnet currents do not have to switch with beam energy. In addition to this, the time for a given magnet to adjust and stabilise must reduce with the time taken to deliver the beam ie. by a factor of a thousand compared to presentday treatment rates. In this respect the development of continuous scanning is of interest [14], since this will eliminate a large fraction of the dead time between spots. Again, the dose monitoring requirements are stringent: however, unlike a passively-scattered system, one is no longer monitoring the current of the entire beam but must monitor spot by spot. It should be noted that these requirements are common across all clinical spot-scanning systems, irrespective of the accelerator technology that drives them.

Clearly, for a spot-scanning system the total number of spots and energy layers is critical in establishing a baseline for the overall speed of the system. Due to the open questions about how FLASH treatment might be delivered, delivery of a single $4 \mathrm{~Gy}$ fraction applied using a single field is assumed; multiple-field treatments are expected to reflect the issues seen in single-field delivery. In order to provide greater spot and layer switching time, an optimised treatment plan for the 1 litre volume was recalculated using the method described in [14]. This spotreduced "van de Water" plan reduced the number of energy layers to 16 , with a maximum number of spots in any layer of $310,{ }^{2}$ partly through variations in the beam spot size from $2-20 \mathrm{~mm}$. A longitudinal slice through the resulting dose distribution of this spot-reduced plan is shown in Fig. 1. The total number of protons was reduced slightly from 3.8 to $3.3 \times 10^{11}$ when compared to the "Gräff" plan introduced in Section 2 , thereby reducing the average current for a $40 \mathrm{~Gy} / \mathrm{s}$ dose rate to a 1 litre volume to $520 \mathrm{nA}$. The differences between the three calculated treatment plans are summarised in Table 2.

In combination with a large energy acceptance, a very fast change of energy could be faster than the lateral shift of the pencil beam. This can be achieved by using by a rapidly-adjusting degrader or by using a ridge- or pin-filter (see Section 5.2). As will be clear from the discussions on lateral scanning speed in the next sections, use of such a filter would enable other sequences in the dose delivery process, such as for example column painting instead of layer painting.

\footnotetext{
${ }^{2}$ Spot reduction treatment planning calculation carried out by Steven van de Water, PSI [14].
}

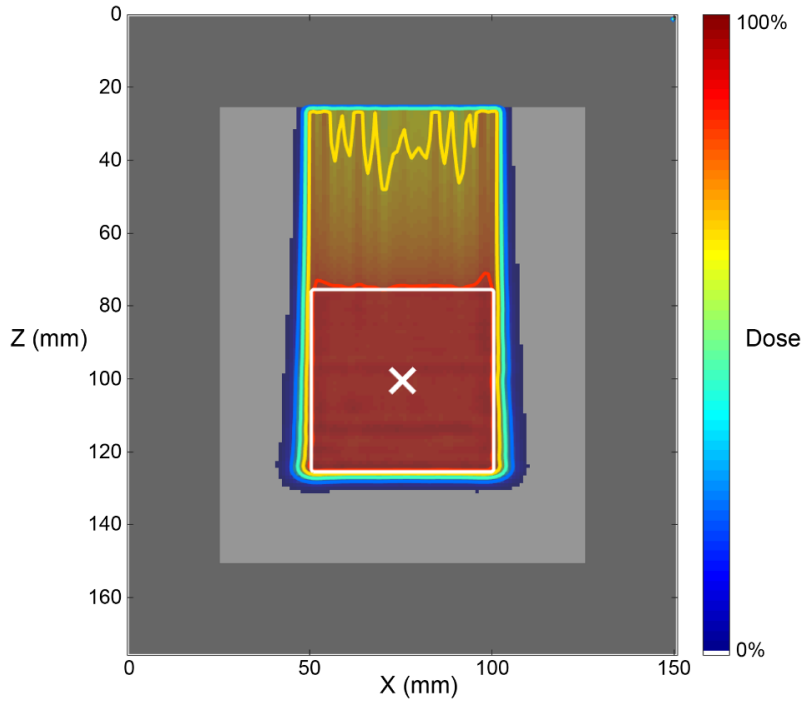

Fig. 1. Longitudinal dose distribution (in $z$ ) for the central slice of the spotreduced "van de Water" treatment plan [14]; beam enters from above. The colours indicate the fraction of the maximum dose within the plan. Note the dose uniformity within the treatment volume despite the reduction in number of spots by 2 orders of magnitude.

\subsection{Magnet switching}

In existing clinical Treatment Planning Systems (TPS) the energy layer spacing is on the order of $3 \mathrm{~mm}$ : for the standard 1 litre volume this corresponds to $\sim 30$ energy layers. To deliver the full volume at FLASH rates the accelerator system must therefore deliver a single layer, then adjust the energy and be ready to deliver the subsequent layer in $\sim 3 \mathrm{~ms}$. Using the spot-reduced treatment plan described above, since the number of energy layers is reduced to 16 this time increases to $\sim 6 \mathrm{~ms}$ : this is also true for the 17-layer $125 \mathrm{ml}$ volume. This time will vary slightly, depending upon whether the system is limited by maximum beam current or magnet switching time: since somewhere around 10 times more protons need to be delivered for distal than proximal layers, the distal layer can either take longer to deliver than the proximal layer in the former case or be delivered at a higher current in the latter. The available time budget can be subdivided into 2 parts:

1. Delivery of a single energy layer;

2. Adjustment of energy between layers.

Given that the total time budget per layer is $\sim 6 \mathrm{~ms}$, this implies that each of these can take no more than $\sim 3 \mathrm{~ms}$. One can envisage that, whilst the accelerator is adjusting the beam energy for the next layer, the beamline is also preparing for the delivery of this new energy. As such, for a system using conventional beamline optics, each of the beamline magnets - quadrupoles, dipoles etc. — must be able to adjust to and stabilise at the correct field settings in around a millisecond. This is not beyond the realms of existing technology given that, for example, the ISIS Rapid Cycling Synchrotron (RCS) ramps up in energy at a rate of $\sim 70 \mathrm{MeV} / \mathrm{ms}[48,49]$. However, the difference for a clinical beamline is that the magnets must adjust and then stabilise rather than cycling continuously over a range of fields. An alternative would be to use a beamline with a suitably wide energy acceptance such that all magnetic fields vary at the same speed during beam delivery, or such that the magnets could be set to accept the complete range of energies for a given treatment plan. Such designs have already been investigated for compact, large energy acceptance gantries $[50,47]$ but prototype clinical systems have not yet been realised.

For a scanning system the most severe requirements are those placed on the nozzle scanning magnets. For the aforementioned 1 litre 
water volume, a typical layer contains 2,500 spots in a $50 \times 50$ grid pattern; for the $5 \times 5 \times 5 \mathrm{~cm}^{3} 125 \mathrm{ml}$ volume all bar the proximal layer contain 625 spots in a $25 \times 25$ grid pattern; for the spot reduction plan, each layer contains at most 310 spots: the spot array is, however, distinctly non-uniform, with gaps between spots adjacent anywhere between 1 and $102 \mathrm{~mm}$. As such, the calculations below are based upon the common minimum spacing of all plans of $2 \mathrm{~mm}$ per spot. For the 1 litre volume, if each layer has to be delivered in $\sim 3 \mathrm{~ms}$, that places a limit of $1.2 \mu$ s for each spot to be delivered and the nozzle magnets to adjust and steer the beam on to the next spot. For the $125 \mathrm{ml}$ volume this time extends to $5 \mu \mathrm{s}$. Assuming a similar time budget for the energy layer adjustment, with a 50/50 split between steering and beam delivery, this requires both the horizontal and vertical scanning magnet to adjust and settle in $\sim 600 \mathrm{~ns}$, or $\sim 2.5 \mu \mathrm{s}$ for the $125 \mathrm{ml}$ volume. This assumes that the scanning system utilises a step-and-shoot approach wherein the beam is switched off entirely between spots. With a gap between spots of $\sim 2 \mathrm{~mm}$, this means a scanning speed of $2 \mathrm{~mm} / 600 \mathrm{~ns}$, or $\sim 3 \mathrm{~m} / \mathrm{ms}$ at isocentre for a 1 litre volume or $\sim 75 \mathrm{~cm} / \mathrm{ms}$ for a $125 \mathrm{ml}$ volume. By comparison, the fastest scanning speed possible on PSI gantry 2 is $2 \mathrm{~cm} / \mathrm{ms}$ [39]. As such, to meet the provisional FLASH specifications requires an increase in scanning speed by 2 orders of magnitude. While magnet systems capable of steering at such speeds already exist in high-energy physics facilities, realising a cost effective clinical system that is able to achieve such a speed, in combination with the stepping process, is extremely challenging.

This requirement can be relaxed slightly if a continuous scanning method is used rather than step-and-shoot, meaning that the beam does not switch off during a single layer scan but is scanned continuously across the energy layer (for a description of this technique, see [51]). For a continuous scan of a 1 litre volume, the scanning speed is reduced somewhat: the beam must scan a total distance of $510 \mathrm{~cm}-50$ lines of $10 \mathrm{~cm}$ each, plus the orthogonal $10 \mathrm{~cm}$ to move lines - which must be covered in $\sim 3 \mathrm{~ms}$, giving a scanning speed of $1.7 \mathrm{~m} / \mathrm{ms}$, which is half the speed requirement of step-and-shoot; for a $125 \mathrm{ml}$ volume this drops further to $43 \mathrm{~cm} / \mathrm{ms}$. However, using a continuous scan places tighter requirements on the stability and monitoring of the delivered dose, since the pencil beam moves faster over the volume. Unless beam current fluctuations occur on much shorter timescales than that for the dose delivery i.e. over nanoseconds, dose errors are more difficult to overcome with continuous scanning, particularly if there are systematic offsets in the dose delivery. Online monitoring and feedback is also more challenging due to the interplay between dose delivery and scanning speed. It also means that spot-reduced plans - such as the one discussed above - cannot be delivered since the spot reduction process necessarily removes certain spots, meaning that the beam must be switched off between spots.

\subsection{Dosimetry}

As noted above, for the step-and-shoot method the time budget for delivering protons to a given spot is $\sim 600 \mathrm{~ns}$ in order to ensure delivery of the standard 1 litre clinical plans within $100 \mathrm{~ms}$. For existing clinical systems with a dose rate of $2 \mathrm{~Gy} / \mathrm{min}$, this time is in the range $1-20 \mathrm{~ms}$, around four orders of magnitude slower. This places severe constraints on the various dosimetry systems that monitor the beam.

At present, the number of protons delivered to a given spot is governed by the dose monitors in the nozzle near the patient. As protons are delivered, these dose monitors record the amount of deposited dose until it has reached the required level, taking account of the delays inherent in the dose monitoring instruments (usually ion chambers). At this point, the signal is sent to the accelerator to switch off the beam. The beam delivery system is then adjusted to prepare for protons to be delivered to the next spot and the process continues. In order to achieve this, the dose monitors need to have the necessary accuracy to ensure the correct dose is delivered within the required tolerance levels: this is normally $<10 \%$, but for FLASH this is not known yet. They also need to be able to respond quickly enough to shut off the beam once the desired dose has been reached for a given spot. In addition, should a safety interlock occur, the beam must be shut off rapidly enough that the patient is not in danger of being overdosed. This means that the dose monitors as well as the associated data-acquisition systems have to be able to monitor considerably faster than the time for delivering the beam to a single spot, so faster than $100 \mathrm{~ns}$.

At such short times, existing ionisation chambers become completely redundant: amongst other issues, the drift time of the ions and electrons within an ionisation chamber is simply too slow to be able to provide continuous sampling of the delivered dose and saturation effects introduce nonlinearities into the ionisation chamber response which are difficult to correct for at extremely high doses [52,53]. As such, new dose monitoring techniques will be required. One possibility is the use of fast scintillators: plastic scintillators have time constants on the order of a few nanoseconds so could provide an alternative. However, the disadvantage of using scintillators is that the light output is no longer a linear function of dose: due to light quenching, the proportion of dose converted to light decreases with higher ionisation density, for example in the Bragg peak. Unlike ionisation chambers, scintillators also "burn out", wherein the light output decreases over time with increased integral dose. It is likely, therefore, that new technology, such as gas scintillation, will be required in order to provide online monitoring of the required dose with a spot-scanning system [54].

The alternative would be to fundamentally change the methodology behind clinical proton beam delivery. At present, the dose is monitored constantly and the amount of protons for each spot "drip-fed" over many pulses until the required dose is reached. If the amount of protons in each pulse can be regulated to within the $10 \%$ tolerance given above, it no longer becomes necessary to deliver protons in this way: the correct amount of protons for each spot is already known a priori by the accelerator. This, however, requires significant improvements in the stability of proton delivery from all existing accelerator systems.

\section{FLASH proton delivery: hybrid systems}

A third possibility that falls somewhere between traditional scanning and scattering methods is to employ a combination of both technologies. There are two possible options:

1. Use a broad beam of a single energy that covers the entire treatment volume. The energy of the broad beam is then varied to cover multiple depths.

2. Use a spot-scanned beam, but employ a range modulator to introduce the required energy spread into the beam, essentially delivering a Spread Out Bragg Peak (SOBP) spot by spot.

It should be noted that neither of these options would be able to utilise the van de Water spot reduction method mentioned above [14], since both methods require a contiguous irradiation field. As such, the accelerator and beamline requirements for each method are based on the proton rates given in Section 2 .

\subsection{Hybrid delivery: broad beams}

Option 1 requires a beam that is larger than the given field size and is therefore similar to traditional scattering systems. However, in order to deliver a conformal dose, the energy of the beam not only needs to be varied but the beam size also needs to be adjusted to match the transverse size of the tumour at each energy layer. This requires the use of a suitable MLC that adjusts the transverse size of the beam to match the tumour at the given energy layer. Based on the values given at the beginning of Section 4.1, wherein the budget for layer delivery and switching is $3 \mathrm{~ms}$, the MLC needs to adjust and settle in $\sim 1 \mathrm{~ms}$. This is not possible with existing MLC systems used in conventional (photon) radiotherapy linacs, and it is difficult to envisage how such a 
specification might be achieved. It should also be noted that there are no MLCs in clinical operation for proton therapy. As with spot-scanning systems, this option also requires energy variation at the same speed as the MLC variation.

The advantage, however, is that dose no longer needs to be measured for an individual spot but instead for an entire layer. This means dose can be measured at similar speeds to present spot-scanning systems, i.e. on the order of $1 \mathrm{~ms}$ for total dose delivery. Although the maximum dose to be monitored is 2,500 times higher, both the dynamic range and the speed of the dose monitors are similar to existing requirements.

\subsection{Hybrid delivery: spot scanning}

In option 2, the opposite approach is chosen: the beam is now scanned across the target but an energy variation is introduced such that all energy layers are delivered simultaneously [56]. Clearly, in order to get accurate scanning, this energy spread must be introduced after the scanning magnets in order that the scanned beam reaches the correct target volume in the tumour: this is currently achieved with 3Dprinted range modulators [55]. An example of such a range modulator is shown in Fig. 2. The range modulator sits next to the patient and consists of a series of vertical pyramid-shaped pins with the same transverse dimensions of an individual spot that introduce an energy spread into the beam. This energy spread can be customised for each spot by tailoring the height of each pin. Experimental facilities have been designed that employ this technique [57]. This method has the advantage that the energy adjustment from the accelerator - and therefore any corresponding magnetic adjustment - is completely removed. As such, so long as the time structure of the beam is suitable, any of the available accelerator technologies can deliver the beam. Notwithstanding the scanning magnets themselves, the magnetic beamline is also completely static during delivery of a single field. Compared with a pure spot-scanning system, the required scanning speed for a 1 litre volume is significantly reduced: with 2,500 spots delivered in $\sim 100 \mathrm{~ms}$, the spot dwell time would be $\sim 20 \mu$ s with $\sim 20 \mu \mathrm{s}$ to steer the beam between spots. For a $125 \mathrm{ml}$ volume this scanning speed reduces further, with both a dwell and steering time of $\sim 80 \mu \mathrm{s}$. This is still quite challenging but less so than a pure spotscanning system.

The disadvantage of a 3D-printed range modulator is that, as with the absorbers and collimators needed for conventional double-scattered proton therapy, one must be printed for every field that is delivered to every patient. In the case of multiple fractions, if the treatment plan needs to be adjusted between fractions then new range modulators are also needed. In addition, the material needs to be selected carefully to prevent significant additional neutron dose to the patient. Finally, compared to option 1 the dosimetry requirement becomes considerably

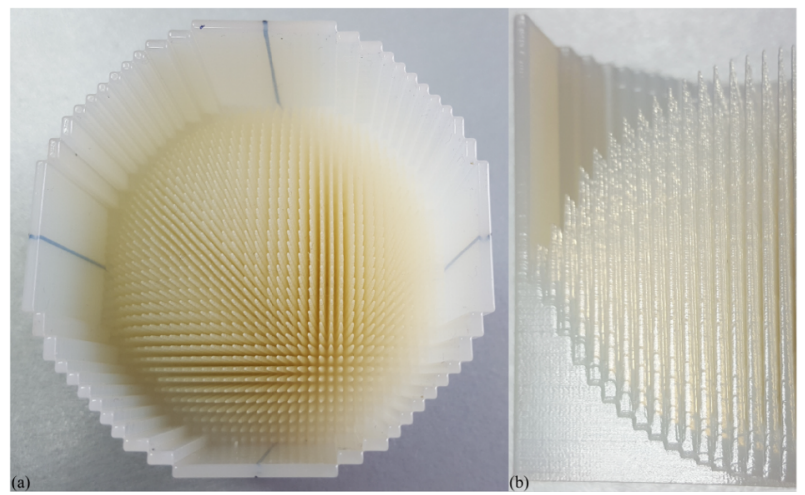

Fig. 2. Example of a 3D range modulator; top view (a) and a quarter of it, side view (b); image taken from [55]. Diameter $d=5 \mathrm{~cm}$, height $h=4.6 \mathrm{~cm}$. more challenging, since the dose must be measured at least 40 times faster for a $125 \mathrm{ml}$ volume and over 70 times faster for a 1 litre volume, but with the same accuracy.

\section{Cyclotrons}

Meeting the challenges and requirements set out in the previous sections places new requirements on the accelerator used for producing the beam. By far the most common accelerator used for existing clinical PBT systems is the cyclotron. The layout of a typical cyclotron-based facility is shown in Fig. 3 Experiments by IBA have been publicised in which small volumes have received FLASH dose rates using a normal conducting clinical cyclotron and scanned-beam delivery [58]. For a $125 \mathrm{ml}$ volume, a dose rate of $45 \mathrm{~Gy} / \mathrm{s}$ delivered in $540 \mathrm{~ms}$ was achieved; for a smaller $2 \times 2 \times 2 \mathrm{~cm}^{3}$ volume a rate of $200 \mathrm{~Gy} / \mathrm{s}$ delivered in $170 \mathrm{~ms}$ was observed [58]. While these experiments do not yet meet the dose rate and delivery time for the 1 litre standard defined in Section 2, they clearly demonstrate the potential for cyclotrons to deliver FLASH proton therapy.

Since a cyclotron produces a continuous beam of high intensity at a fixed energy, a degrader is necessary to produce the necessary variation in energy. One method to achieve fast intensity variation is by means of beam intercepting systems in the central region of the cyclotron, where the beam energy is still low enough to prevent activation and cooling problems. Present-day typical maximum extracted beam intensities from a proton therapy cyclotron are in the range $100-800 \mathrm{nA}$. More modern ion source designs and central region layouts could perhaps increase the extracted current by a factor of ten, thereby meeting the continuous current requirement for FLASH (see Section 2). For cyclotrons the biggest challenges in delivering FLASH with a cyclotron are related to beam energy. Beam energy cannot be modified very rapidly in a cyclotron: the beam energy is controlled by a degrader in the beam transport system that introduces a variable ionisation loss by varying the physical thickness and/or density of inserted degrader material. For FLASH systems which require energy variation from the accelerator, the degrader should be able to change the beam energy very fast - perhaps $<0.1 \mathrm{~ms}$ /energy step - which is challenging. However, when it is mounted at a location where the beam cross section is only a few millimetres, such as a beam-optical waist, the design can benefit from the small distances to be covered to insert material into or out of the beam path.

A major disadvantage of a degrader is the scattering that accompanies the ionisation loss that leads to an increase of the beam emittance and energy spread; this effect becomes very serious at lower energies. The characteristics of the desired dose distribution at the patient determine the trade-off between the emittance and energy spread chosen from the exit distribution (by means of an energy selection system and collimators) and the obtained current and spot size at the patient. For example, at the PSI PROSCAN facility the beam spot size is kept reasonably small (less than $10 \mathrm{~mm}$ ) even at the lowest, most-degraded energies, and with an energy spread less than $1 \%$; the consequence at $70 \mathrm{MeV}$ is an overall transmission of only $\sim 0.1 \%[59,60]$ : more than $99 \%$ of the beam extracted from the cyclotron is lost, which places a lower limit on the energy that (high-energy) cyclotrons can deliver with an acceptable intensity. Typical intensity reductions cause by a cyclotron ESS are shown in Fig. 4. Improved degrader geometries may help somewhat $[60,61]$, but it also suggests the need for dedicated lower-energy intense cyclotrons that avoid the need for some of the degrader material. Should a single cyclotron be used to cover all the clinical energy ranges, and assuming the existing transmission loss of $99 \%$ at low energies, currents approaching $100 \mu \mathrm{A}$ will need to be extracted to meet the requirements for the 1 litre volume set out in Section 2. Such currents are achievable with cyclotrons, but are two orders of magnitude above the limits of existing clinical systems. As such, adaptation of high current cyclotron designs will be necessary in order to realise a clinical FLASH system with full spot scanning. It is likely, 


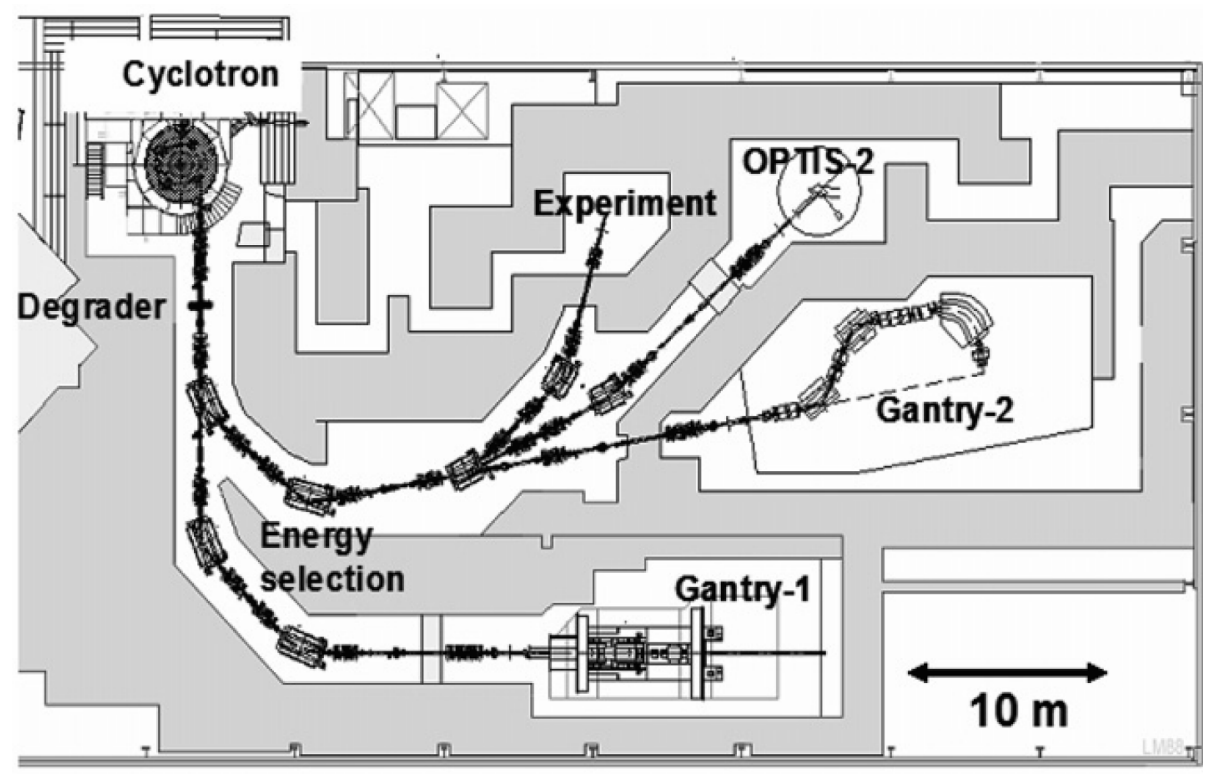

Fig. 3. Layout of the PSI PROSCAN cyclotron-based proton therapy facility; taken from [20].

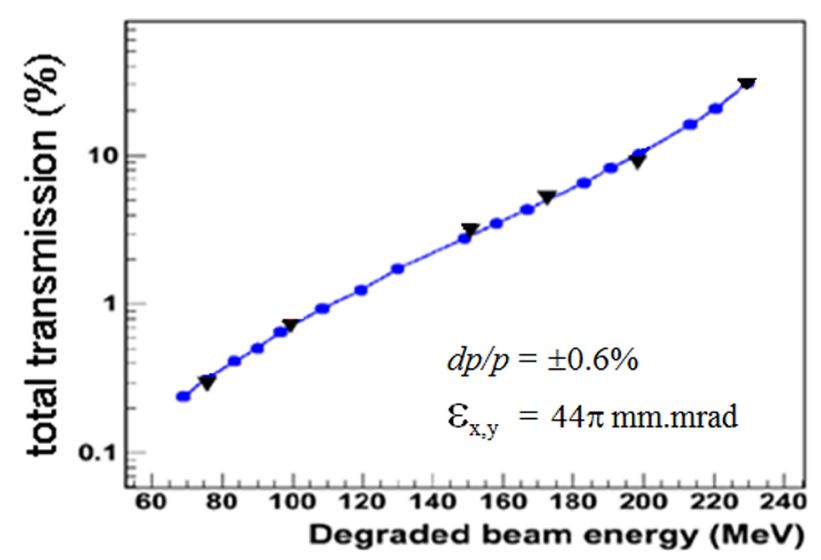

Fig. 4. Example of typical transmission with energy through a cyclotron degrader and ESS: the Paul Scherrer Institute PROSCAN facility; figure reproduced from $[60]$.

therefore, that the first clinical FLASH systems will utilise cyclotrons delivering beam via the hybrid systems described in Section 5.2.

Intensity regulation can be carried out quickly and accurately by beam manipulation in the central region (i.e. in the first turns) of a cyclotron. However, this will need to be coupled with suitably fast monitoring and signalling at an appropriate location in the clinical beamline to ensure the beam delivered to the patient is also cut off suitably quickly. In addition to the time response of the signaling detector, there is also the role played by the signal transfer time between the detection equipment at the steerer/interceptor within the cyclotron. The typical distances traversed by signals in a facility correspond to propagation delays of several hundred nanoseconds, which acts as an inherent lower limit on the beam interruption response time in the absence of predictive dose delivery, regardless of the detector type. Although this propagation delay corresponds to $10^{-4}$ of the total $100 \mathrm{~ms}$ dose delivery, it can contribute significantly to the dose error in those parts of the target volume when the beam shut-off is initiated.

\section{Synchrotrons}

Synchrotron-based systems for PBT are less common than cyclotronbased systems due to their increased complexity. The layout of the MedAustron synchrotron-based facility is shown in Fig. 5. They have the advantage that the extracted energy can be preselected by the accelerator rather than requiring an external degrader. However, most present-day synchrotrons perform the dipole ramping at relatively slow rates; typically, a full dipole cycle - from injection energy up to extraction and then back down again - takes a few seconds. Another limitation is that the majority of synchrotrons store only a single bunch

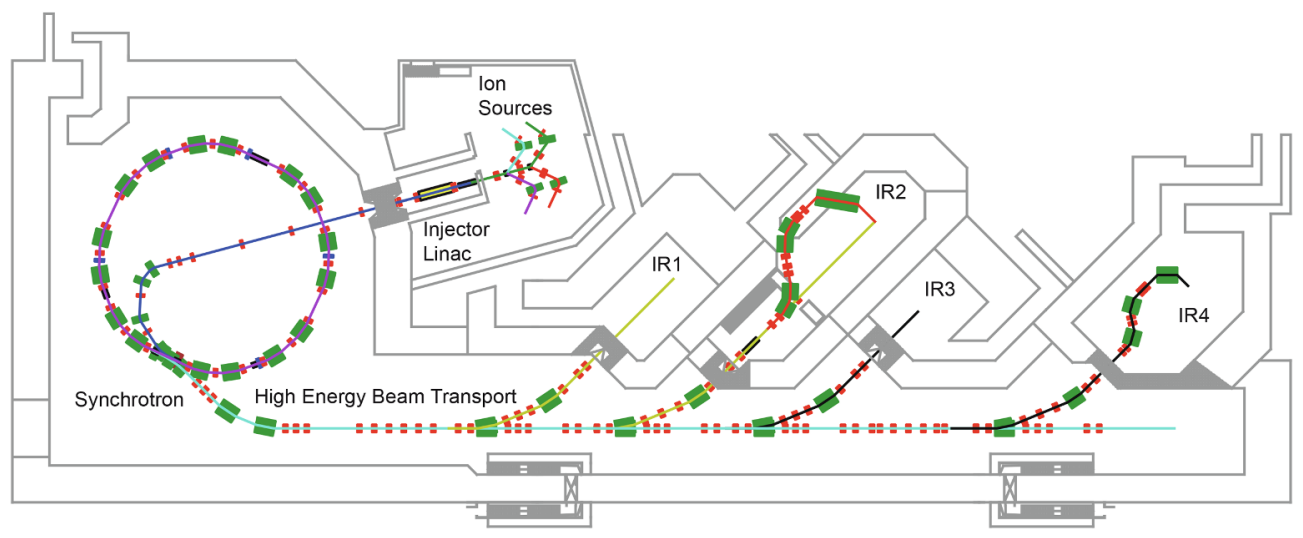

Fig. 5. Layout of the MedAustron accelerator and beamlines, including the four Irradiation Rooms (IR); adapted from [62]. 


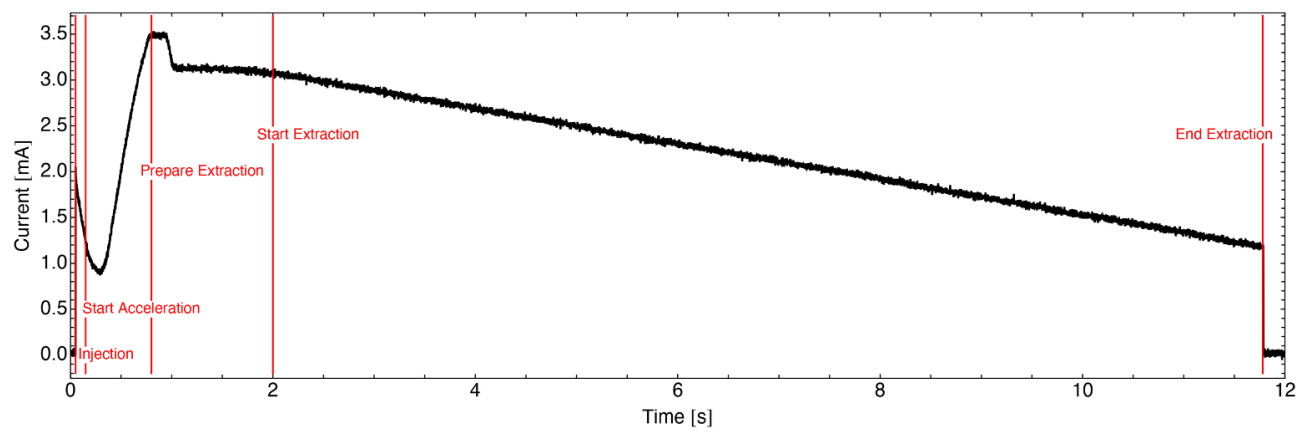

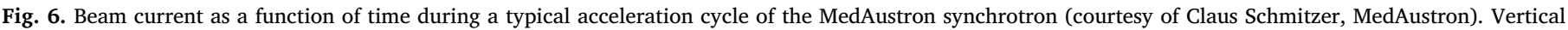
lines indicate the key stages in the acceleration and extraction cycle.

of protons, which through space-charge limits and dipole cycle rate effectively limits the average dose rate that may be delivered to a patient treatment. A single injected bunch from a linear pre-accelerator is accelerated and then generally slowly extracted to deliver a stable dose rate to the patient. The beam current within a typical medical synchrotron ring is shown in Fig. 6: note that the acceleration cycle takes less an a second but that the extraction of the beam takes nearly $10 \mathrm{~s}$ before the unwanted protons are dumped.

In order to realise FLASH proton therapy with a synchrotron several technical challenges need to be overcome, some of which involve significant design and operational changes. FLASH proton delivery will need to take advantage of multi-energy extraction: rather than dumping the remaining protons within the synchrotron ring after a single energy has been delivered, the remaining protons are re-accelerated or decelerated to match the next energy layer, thereby removing the need to re-inject fresh protons and accelerate from the low injection energy. This technique has been utilised for clinical treatment at HIMAC [63] and has been implemented and demonstrated with the Hitachi commercial system [27]. However, this has been limited to a few energy layers, not an entire treatment, with energy adjustment between layers taking around $30 \mathrm{~ms}$. To support this, a larger stored proton current within the synchrotron will also be necessary.

\subsection{High-current injection and storage}

Obtaining a high intensity requires a large bunch current; the present maximum bunch charge in medical synchrotrons is $\sim 10^{12}$ protons: while this is above the $4 \times 10^{11}$ required for the 1 litre $40 \mathrm{~Gy} / \mathrm{s}$ FLASH delivery (see Section 2), it doesn't account for proton losses during beam extraction and delivery. This is largely a result of space charge limitations: increasing this will likely require a higher injection energy. This is not a technical limitation; linear accelerators exist in high-energy physics - such as Linac4 at CERN - that can accelerate orders of magnitude more protons to well above $100 \mathrm{MeV}$. This is a question of cost: increasing both the final energy and the transmitted current from the linac will result in a significant cost increase.

\subsection{Energy variation}

Assuming a large stored current is possible, a single dipole cycle could be utilised, meaning that multiple injection cycles are no longer necessary. However, in this case rapid multi-energy extraction is needed, covering the entire treatment volume rather than a subset of layers as is presently done. To meet the speed requirements for the full spot-scanning delivery of the 1 litre volume set out in Section 4 , the synchrotron will need to adjust the beam energy and extract all the protons necessary for a given energy layer in $\sim 3 \mathrm{~ms}$ : this rises to $6.25 \mathrm{~ms}$ when using the spot-reduced plan. This implies that the longest a synchrotron can take to go from a stable circulating beam at one energy to a stable circulating beam at another energy is $\sim 3 \mathrm{~ms}$ : this is at least an order of magnitude faster than is currently possible. Assuming an energy step of $\sim 1.5 \mathrm{MeV}$ (see Section 2) this implies an energy change $\sim 0.5 \mathrm{MeV} / \mathrm{ms}$. Such a rate is not outside the capabilities of existing synchrotrons: Rapid Cycling Synchrotrons (RCSs), such as the ISIS spallation neutron source [64], are capable of accelerating at rates above $70 \mathrm{MeV} / \mathrm{ms}$ [49].

\subsection{Rapid extraction}

Finally, assuming that it is possible to inject a suitable number of protons for the entire treatment into the synchrotron and adjust the energy for consecutive layers faster than $\sim 0.5 \mathrm{MeV} / \mathrm{ms}$, stable extraction of all protons necessary for a single energy layer must be possible in $<3 \mathrm{~ms}$. The two most common methods for extraction from clinical synchrotrons are via RF knockout or betatron core extraction [65,66]. Both methods are intended to extract the beam slowly over the course of several seconds. RF knockout provides more stable extraction (albeit with variations that must be accounted for [67-69]) that can be switched on and off more quickly; systems utilising a betatron core need another device to deflect the beam downstream of the extraction point in order to switch the beam off between spots.

To provide the temporal beam characteristics needed for FLASH spot scanning (see Section 4.1), the extraction time for a single energy layer will need to be reduced from several seconds to $<3 \mathrm{~ms}$, a reduction by 3 orders of magnitude. This clearly requires a novel approach not yet described. The large variations in current during extraction also need to be significantly reduced - ideally below $10 \%$ - in order to prevent under- or overdosing of individual spots. Ideally this new extraction method would have rise and fall times $\ll 600$ ns to allow the beam to be switched off between spots when using the step-and-shoot method. This condition relaxes by a factor of two if using a continuous scan, but doing so places tighter constraints on the temporal stability since the beam cannot be switched on and off to deliver the correct dose for a given spot.

The extracted pulse structure has a further implication for the RF frequency used for bunching and accelerating the beam. In present systems this is between $2-6 \mathrm{MHz}$, depending upon the size of the synchrotron and the extraction energy. However, as described in Section 4.1 , the time to deliver a given spot is $<600 \mathrm{~ns}$ for the clinical standard 1 litre volume or $<2.5 \mu$ s for the $125 \mathrm{ml}$ volume. For the larger volume the number of protons extracted during a given turn must therefore be correct for a given spot, rather than being slowly accumulated; for the smaller volume far fewer turns would be available to accumulate the necessary dose compared to conventional proton therapy. In addition, the magnet switching time must be matched to the bunching frequency. An alternative would be to bunch and accelerate the beam at a much higher frequency - closer to $100 \mathrm{MHz}$, the frequency regime of cyclotrons - enabling protons extracted from several bunches to be delivered to a single spot. This has the disadvantage that the natural gap between bunches provided by the bunch structure within the ring 
becomes much shorter, making it more difficult to use this for switching the beam off between spots.

These extremely challenging requirements become relaxed somewhat when considering the hybrid systems described in Section 5. When using the broad beam option (see Section 5.1), the individual spot requirements are removed. The $10 \%$ stability requirement is also only necessary over one milliscond, rather having to be achieved in a microsecond. However, the need for multi-energy extraction is still present with its associated temporal requirements. For the scanned beam hybrid option (see Section 5.2), with a spot delivery time of $20 \mu \mathrm{s}$ the interplay with the bunching frequency is no longer such an issue. In addition, multi-energy extraction is no longer required since only a single energy needs to be extracted from the synchrotron. The requirements are still extremely challenging compared to existing systems, since the spill length can be no longer than $100 \mathrm{~ms}$ and the extraction must be stable to $10 \%$ within the delivery time for each "spot" of $20 \mu \mathrm{s}$. Even more so than with cyclotrons, new technological advances will be required in order to realise FLASH delivery with synchrotrons. Some possibilities may exist for synchrotrons with fast (ie. single turn) extraction in combination with a passive dose delivery system.

\section{Synchro-cyclotrons}

Most of the cyclotrons used in current PBT facilities are isochronous cyclotrons, which produce a effectively continuous current since their extraction pulse rate is typically $30-70 \mathrm{MHz}$ (matching the constant RF at the accelerating Dees). Synchro-cyclotrons have also been introduced into modern proton therapy, and can offer a more compact footprint by using a higher average magnetic field above $4 \mathrm{~T}$ and even up to $9.4 \mathrm{~T}$ $[70,71]$. Synchro-cyclotrons utilise a varying Dee frequency which only a single accelerated bunch can be synchronous to, and are limited by the RF system to extracted bunch frequencies no more than about $1 \mathrm{kHz}$; the intensity will therefore be limited by space charge and possible beam losses at extraction near superconducting coils. For example, the IBA S2C2 5.7T synchro-cyclotron delivers a repetition rate of $1 \mathrm{kHz}$ with a maximum extracted current above $100 \mathrm{nA}$ and a $7 \mu \mathrm{s}$ long pulse [70]: the layout of the IBA S2C2-based Proteus One system is shown in Fig. 7. For the clinical specification given in Section 2, only 100 pulses would be possible within the required $100 \mathrm{~ms}$ time window. This limits synchro-cyclotrons utilising step-and-shoot spot scanning to volumes around $1 \mathrm{~cm}^{3}$, assuming that the number of protons in a given pulse can be controlled accurately enough to give the correct dose to a single spot in a single pulse.

Although possibilities may exist for synchro-cyclotrons in combination with a passive dose delivery system, for those delivery techniques that require energy variation from the accelerator, such as spot scanning, this implies delivering one-third of each energy layer of a 1 litre volume within in $7 \mu$ s unless there is a way to lengthen the pulse or increase the repetition rate. This makes a pure spot-scanning approach with a synchro-cyclotron more difficult than for an isochronous cyclotron. For the $125 \mathrm{ml}$ and spot reduction plans outlined in Section 4 - with half the number of energy layers - one-sixth of each layer will need to be covered within each $7 \mu$ s pulse. Since this implies covering several spots with a single pulse, continuous scanning will be necessary. A faster repetition rate would relax these constraints, but technical limitations inherent in the synchro-cyclotron design rule out repetition rates much above the $1 \mathrm{kHz}$ used in existing systems.

Initial experiments have been publicised in which small $\left(2 \times 2 \times 2 \mathrm{~cm}^{3}\right)$ volumes have received FLASH dose rates in excess of $60 \mathrm{~Gy} / \mathrm{s}$ using scanned-beam delivery from a clinical synchro-cyclotron [73]. Extending this to larger treatment volumes will require either increased extracted current - which appears to be feasible up to perhaps $150 \mathrm{~Gy} / \mathrm{s}$ - or to deliver FLASH doses separately to different parts of a treatment volume, in other words not treat the whole volume at the same time.

The hybrid systems presented in Section 5 provide alternative routes to clinical delivery, although challenges remain. The broad beam option may be more feasible if enough current can be delivered in each pulse to fully cover each layer in 3 pulses. For the scanned beam option, the scanning speed is more challenging for the 1 litre volume since, rather than being able to make a continuous or step-and-shoot scan within the required $100 \mathrm{~ms}$ time window, the pulsed synchro-cyclotron beam must cover $1 \%$ of the target volume within $7 \mu \mathrm{s}$. For the clinical volume defined in Section 2, this means scanning 25 spots in $7 \mu$ s or 280 ns per spot: this is twice as fast as the requirement for a spot-scanning system utilising a continuous rather than a pulsed beam (see Section 4.1). This is not possible with current technology and makes it unlikely that synchro-cyclotrons would be the eventual technology of choice for a pure spot-scanning FLASH proton therapy system. Assuming that the $100 \mathrm{~ms}$ delivery window cannot be relaxed in order to achieve FLASH effects, if utilising the hybrid scanning modality described in Section 5.2 synchro-cyclotrons would still be limited to 100 spots, so arrays of $10 \times 10$ columns, or scanning areas of $\sim 2 \times 2 \mathrm{~cm}^{2}$, as in the IBA experiments [73]. Controlling the number of protons within each pulse to match the required dose for each column within the required tolerance would also be a necessary technological development, something that is not presently implemented.

\section{Linear accelerators}

Assessing the feasibility of pure linear accelerator-based systems for delivering FLASH proton therapy is more difficult than the other accelerator technologies already mentioned because there are no such clinical systems presently in operation. A notable development is the LIGHT system of AVO-ADAM, which utilises $3 \mathrm{GHz}$ high-gradient accelerating structures to obtain protons up to $230 \mathrm{MeV}$ in $\sim 2 \mu$ s pulses at up to $200 \mathrm{~Hz}$ [29]: the intended layout for this system is shown in

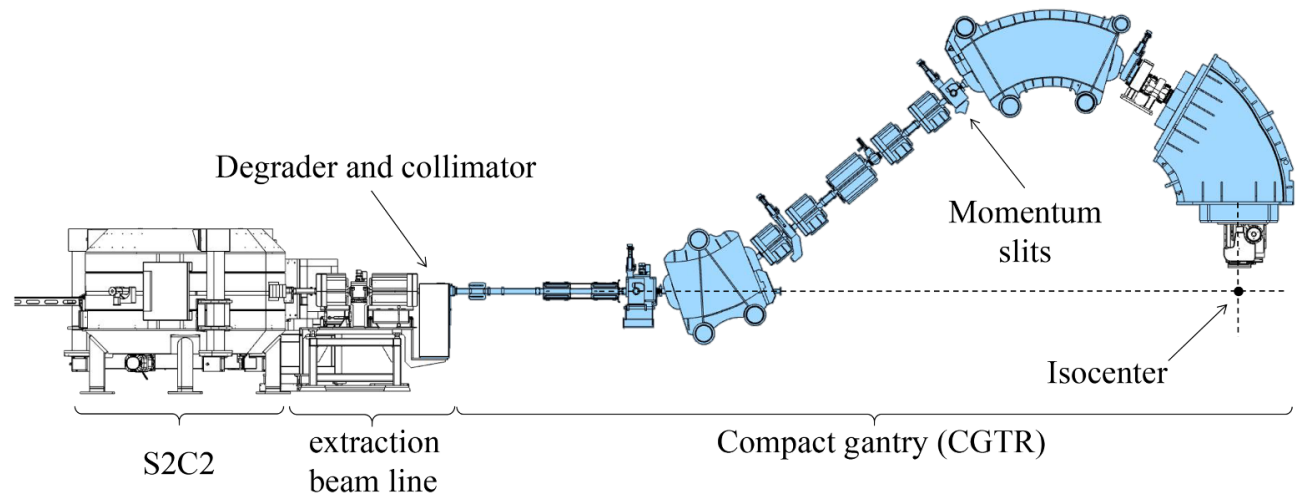

Fig. 7. The IBA Proteus One compact proton therapy machine, which includes the superconducting synchro-cyclotron S2C2; adapted from [72]. 


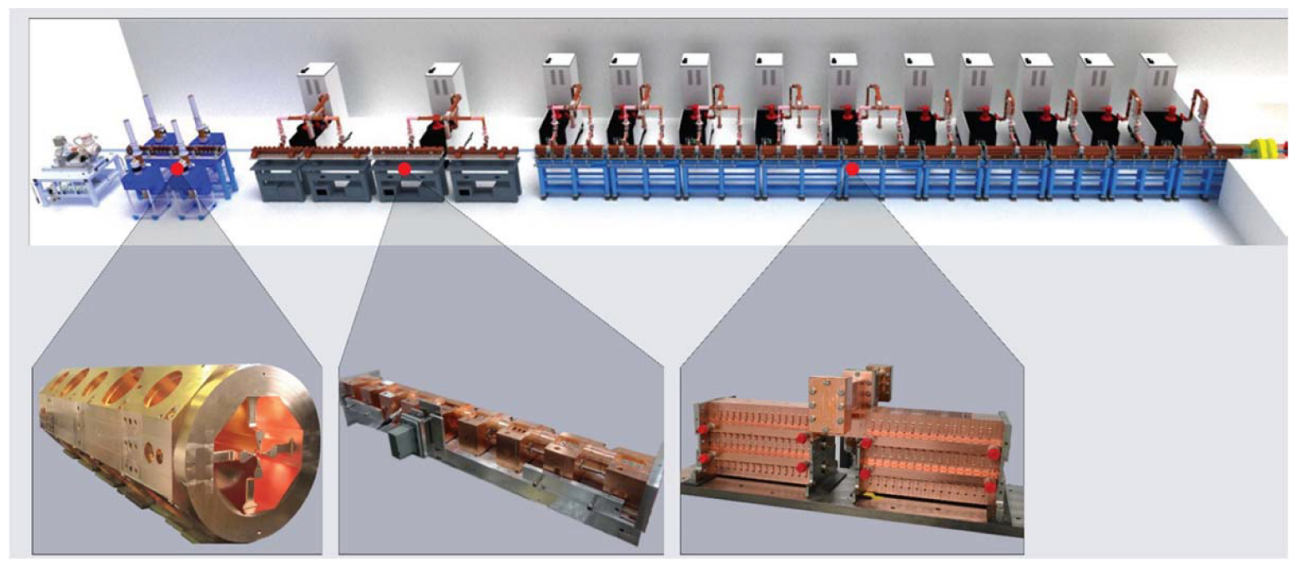

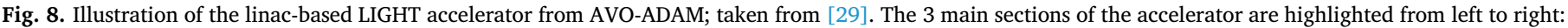
RadioFrequency Quadrupole (RFQ), a Side-Coupled Drift-Tube Linac (SCDTL) and a Cell-Coupled Linac (CCL).

Fig. 8. These bunch timescales are therefore quite similar to those of synchro-cyclotrons.

The advantages of linac-based systems are the higher peak current and smaller beam emittance, coupled with the ability to vary the delivered energy pulse by pulse; no degrader is required, dispensing at low proton energies with the requirement for large accelerated currents and significant degrader losses [74]. However, a $200 \mathrm{~Hz}$ repetition rate gives only 20 separate delivered energy layers within the $100 \mathrm{~ms}$ time window specified in Section 2: this makes a full linac-based spotscanning FLASH system unfeasible without increases in repetition rate of several orders of magnitude to enable repetition rates above $1 \mathrm{MHz}$.

A recent planning study by AVO examined delivering FLASH dose rates within $0.5 \mathrm{~s}$ to $\sim 1 \mathrm{~cm}^{3}$ volumes; mean dose rates up to $93 \mathrm{~Gy} / \mathrm{s}$ were predicted for overall doses of $20 \mathrm{~Gy}$ [75]. Delivering such subsecond doses to larger volumes will likely limit linacs to a broad-beam hybrid approach (see Section 5.1). For the example spot-scanned hybrid setup described in Section 5.2, 2,500 spots would have to be delivered to a 1 litre volume in $100 \mathrm{~ms}$, implying a repetition rate of $25 \mathrm{kHz}$ : ion sources and linacs that function at such high rep rates do not currently exist. It would also require the number of protons within each pulse to be accurate to $10 \%$ with a dynamic range large enough to cover all energy layers: this is extremely challenging.

As such, a linac-based FLASH proton therapy system is less attractive than those based on continuous beam delivery, such as cyclotrons. As mentioned in Section 7.1, where linacs hold an advantage is in peak current, where the space charge limitations of circulating beam accelerators are no longer a factor. As such, should the clinical evidence point towards dose rates well above the present minimum of $40 \mathrm{~Gy} / \mathrm{s}$ but within a delivery window longer than $100 \mathrm{~ms}$ - for example, in the circumstance where smaller volumes can be delivered at FLASH rates within a larger treatment volume - pulsed beams with high peak current become more attractive. This would open the door to FLASH delivery with linacs and synchro-cyclotrons.

\section{Conclusions}

Developing accelerator technology to meet the requirements for FLASH proton delivery is challenging. Whilst experiments have already been carried out with protons at FLASH dose rates [11-13,58,73], these have been limited so far to small volumes. Significant development will be required to enable FLASH delivery beyond these limitations, particularly if the goal is to adapt existing spot-scanning systems for use at FLASH dose rates to clinically relevant volumes: as described in Section 4 , developments in magnet scanning speed and dosimetry will be necessary before such systems can be realised. The hybrid approaches already being pursued - particularly the use of scanned beams with patient-specific range modulators - are likely to pave the way to clinical proton FLASH delivery. Each accelerator type has its own challenges in order to meet this goal.

Isochronous cyclotrons. Existing clinical systems offer beam currents up to $800 \mathrm{nA}$, with research systems in existence capable of producing currents above $100 \mu \mathrm{A}$. The key challenge for cyclotrons is the development of lower loss degraders and energy selection or large acceptance beam delivery, thereby reducing the requirement on maximum extracted current.

Synchrotrons. Whilst the developmental challenges for FLASH systems based on synchrotrons appear daunting, none preclude the development of synchrotrons for FLASH. Linacs that can deliver the necessary peak current and synchrotrons capable of accelerating that current are already in existence, albeit not in clinical systems. The area that will require the greatest adaptation is in matching the extracted beam characteristics to that necessary for FLASH: this will necessitate staged acceleration of the stored protons to multiple energies without the need for re-injection and extraction of the resulting beam with a stability and pulse structure necessary for clinical FLASH delivery. With suitable increases in stored current and spot-scanning speed, FLASH proton therapy may be possible using existing synchrotrons coupled to the hybrid technology described in Section 5.2.

Synchro-cyclotrons. Although some of the first FLASH proton accelerator experiments have been carried out with synchro-cyclotrons [73], hard technical limitations are likely to prevent their use in fully fledged spot-scanning FLASH systems. Primarily, the repetition frequency and pulse structure of synchro-cyclotrons is much less wellmatched to FLASH beam delivery requirements than the stable CW beams produced by isochronous cyclotrons. Should "localised FLASH" be possible, wherein small subvolumes are treated at FLASH rates without the entire volume needing to be treated within $100 \mathrm{~ms}$, synchro-cyclotron-based FLASH systems become more feasible. It is likely, however, that it will be necessary to utilise some form of hybrid scanning.

Linacs. Even more so than for synchro-cyclotrons, the feasibility of developing linac-based FLASH proton therapy systems is highly dependent on the eventual clinical requirements necessary for realising the FLASH effect. At present, the repetition rate is simply too slow to enable delivery to anything but small volumes: at an existing repetition rate of $200 \mathrm{~Hz}$, an increase in this rate by a factor of $>500$ would be necessary for spot-scanned FLASH delivery to a 1 litre volume, or of $>100$ to enable the hybrid delivery described in Section 5.2. Pulsed beams are strongly biased towards high instantaneous current with longer delivery times: should dose rates in excess of $40 \mathrm{~Gy} / \mathrm{s}$ be necessary but without the limitation to deliver the entire dose in $100 \mathrm{~ms}$, synchro-cyclotrons and linacs become more attractive.

Amongst current technologies, the most direct route to a clinical FLASH proton system appears to be with an isochronous cyclotron 
utilising a hybrid spot-scanned approach. Significant changes in the mode of operation or treatment using synchrotrons, synchro-cyclotrons and linacs will be needed in order to enable a fully-fledged FLASH system. However, to design optimal technology for a FLASH system, more knowledge is needed on the biological and clinical issues, most importantly in the areas of minimum dose rate and maximum delivery time. Only with sufficient knowledge of FLASH processes could one exploit a possible NTCP reduction to allow certain technical compromises to relax the constraints on the accelerator technology.

\section{Acknowledgements}

The authors are extremely grateful to Christian Gräff of GSI for calculating the treatment plan for a $2 \mathrm{~Gy}$ dose delivered to a 1 litre cubic volume, using the TRiP98 treatment planning software [33,34]; and to Steven van de Water of PSI for calculating the spot reduced plan [14].

The authors also gratefully acknowledge the discussions and input from participants at the 2019 OMA International Conference on Medical Accelerators and Particle Therapy.

Finally, the authors are grateful to Rudi Labarbe and colleagues from IBA for discussions regarding FLASH delivery with synchro-cyclotrons.

This project has received funding from the European Union's Horizon 2020 research and innovation programme under the Marie Sklodowska-Curie grant agreement No 675265, OMA - Optimization of Medical Accelerators.

\section{References}

[1] Baumann M, Peterson C. TCP and NTCP: A basic introduction. Rays 2005;30(2):99-104.

[2] Paganetti H. Relating the proton relative biological effectiveness to tumor control and normal tissue complication probabilities assuming interpatient variability in $\alpha / \beta$. Acta Oncol 2007;56(11):1379-86. pMID: 28918679. doi:10.1080/0284186X. 2017.1371325.

[3] Wilson P, Jones B, Yokoi T, Hill M, Vojnovic B. Revisiting the ultra-high dose rate effect: implications for charged particle radiotherapy using protons and light ions. Brit J Radiol 2012;85(1018);e933-e939. pMID: 22496068. doi:10.1259/bjr/ 17827549.

[4] Durante M, Bräuer-Krisch E, Hill M. Faster and safer? FLASH ultra-high dose rate in radiotherapy. Brit J Radiol 2018;91(1082);20170628. pMID: 29172684. doi:10. $1259 /$ bjr.20170628.

[5] Favaudon V, Caplier L, Monceau V, Pouzoulet F, Sayarath M, Fouillade C, Poupon M-F, Brito I, Hupé P, Bourhis J, Hall J, Fontaine J-J, Vozenin M-C. Ultrahigh doserate FLASH irradiation increases the differential response between normal and tumor tissue in mice. Sci Transl Med 2014;6(245):245ra93. https://doi.org/10. 1126/scitranslmed.3008973. URL.

[6] Vozenin M-C, De Fornel P, Petersson K, Favaudon V, Jaccard M, Germond J-F, Petit B, Burki M, Ferrand G, Patin D, Bouchaab H, Ozsahin M, Bochud F, Bailat C, Devauchelle P, Bourhis J. The advantage of FLASH radiotherapy confirmed in minipig and cat-cancer patients. Clin Cancer Res 2019;25(1):35-42. https://doi.org/10. 1158/1078-0432. arXiv:http://clincancerres.aacrjournals.org/content/25/1/35 full.pdf.

[7] Montay-Gruel P, Bouchet A, Jaccard M, Patin D, Serduc R, Aim W, Petersson K, Petit B, Bailat C, Bourhis J, Bräuer-Krisch E, Vozenin M-C. X-rays can trigger the FLASH effect: Ultra-high dose-rate synchrotron light source prevents normal brain injury after whole brain irradiation in mice. Radiother Oncol 2018;129(3):582-8. https:// doi.org/10.1016/j.radonc.2018.08.016. URL: http://www.sciencedirect.com/ science/article/pii/S0167814018334546.

[8] Montay-Gruel P, Meziani L, Yakkala C, Vozenin M-C. Expanding the therapeutic index of radiation therapy by normal tissue protection. Brit J Radiol 2019;92(1093):20180008. pMID: 29694234. doi:10.1259/bjr.20180008

[9] Schüler E, Trovati S, King G, Lartey F, Rafat M, Villegas M, Praxel AJ, Loo BW, Maxim PG. Experimental platform for ultra-high dose rate FLASH irradiation of small animals using a clinical linear accelerator. Int J Radiat Oncol Biol Phys 2017;97(1):195-203. https://doi.org/10.1016/j.ijrobp.2016.09.018. URL.

[10] Simmons DA, Lartey FM, Schüler E, Rafat M, King G, Kim A, Ko R, Semaan S, Gonzalez S, Jenkins M, Pradhan P, Shih Z, Wang J, von Eyben R, Graves EE, Maxim PG, Longo FM, BWL Jr. Reduced cognitive deficits after FLASH irradiation of whole mouse brain are associated with less hippocampal dendritic spine loss and neuroinflammation. Radiother Oncol doi:10.1016/j.radonc.2019.06.006. URL: http:// www.sciencedirect.com/science/article/pii/S0167814019329421.

[11] Bourhis J, Jeanneret-Sozzi W, Jorge PG, Gaide O, Bailat C, Duclos F, Patin D, Ozsahin M, Bochud F, Germond J-F, Moeckli R, Vozenin M-C. Treatment of a first patient with flash-radiotherapy. Radiother Oncol 2019;139:18-22, fLASH radiotherapy International Workshop. doi:10.1016/j.radonc.2019.06.019. URL: https:// www.sciencedirect.com/science/article/pii/S0167814019329597.

[12] Physics World. FLASH radiotherapy: from preclinical promise to the first human treatment, accessed: 2019-09-10. URL: https://physicsworld.com/a/flash-radiotherapy-from-preclinical-promise-to-the-first-human-treatment/.

[13] Buonanno M, Grilj V, Brenner DJ. Biological effects in normal cells exposed to FLASH dose rate protons. Radiother Oncol doi:10.1016/j.radonc.2019.02.009. URL: http://www.sciencedirect.com/science/article/pii/S0167814019300763.

[14] van de Water S, Safai S, Schippers JM, Weber DC, Lomax AJ. Towards FLASH proton therapy: the impact of treatment planning and machine characteristics on achievable dose rates. Acta Oncol 2019:1-7. pMID: 31241377. doi:10.1080/ 0284186X.2019.1627416. URL: https://www.tandfonline.com/doi/full/10.1080/ 0284186X.2019.1627416.

[15] Patriarca A, Fouillade C, Auger M, Martin F, Pouzoulet F, Nauraye C, Heinrich S, Favaudon V, Meyroneinc S, Dendale R, Mazal A, Poortmans P, Verrelle P, Marzi LD. Experimental set-up for FLASH proton irradiation of small animals using a clinical system. Int J Radiat Oncol Biol Phys 2018;102(3):619-26. https://doi.org/10. 1016/j.ijrobp.2018.06.403. URL: http://www.sciencedirect.com/science/article/ $\mathrm{pii} / \mathrm{S} 0360301618314044$

[16] Beyreuther E, Brand M, Hans S, Hideghéty K, Karsch L, Leßmann E, Schürer M, Szabó ER, Pawelke J. Feasibility of proton flash effect tested by zebrafish embryo irradiation. Radiother Oncol doi:10.1016/j.radonc.2019.06.024. URL: http://www. sciencedirect.com/science/article/pii/S0167814019329640.

[17] Diffenderfer ES, Verginadis II, Kim MM, Shoniyozov K, Velalopoulou A, Goia D, Putt M, Hagan S, Avery S, Teo K, Zou W, Lin A, Swisher-McClure S, Koch C, Kennedy AR, Minn A, Maity A, Busch TM, Dong L, Koumenis C, Metz J, Cengel KA. Design, implementation, and in vivo validation of a novel proton FLASH radiation therapy system. Int J Radiat Oncol Biol Phys 2020;106(2):440-8. https://doi.org/10.1016/ j.ijrobp.2019.10.049. URL: https://www.redjournal.org/article/S0360-3016(19) 34055-6/fulltext.

[18] Vandeplassche D, Beeckman W, Zaremba S, Jongen Y, Tachikawa T. Extracted beams from IBA's C235. In: Proceedings of the 1997 particle accelerator conference (PAC'97), Vancouver, BC, Canada, 12-16 May, vol. 1; 1997. p. 1045-47. URL: https://accelconf.web.cern.ch/pac97/papers/pdf/7W022.PDF.

[19] Petzoldt J, Roemer KE, Enghardt W, Fiedler F, Golnik C, Hueso-González F, Helmbrecht S, Kormoll T, Rohling H, Smeets J, Werner T, Pausch G. Characterization of the microbunch time structure of proton pencil beams at a clinical treatment facility. Phys Med Biol 2016;61(6):2432-56. https://doi.org/10 1088/0031-9155/61/6/2432.

[20] Schippers J, Dölling R, Duppich J, Goitein G, Jermann M, Mezger A, Pedroni E, Reist H, Vrankovic V. The SC cyclotron and beam lines of PSI's new protontherapy facility PROSCAN. Nucl Instrum Methods Phys Res Sec B: Beam Interact Mater Atoms 2007;261(1):773-6. the Application of Accelerators in Research and Industry. doi:10.1016/j.nimb.2007.04.052. URL: http://www.sciencedirect.com/ science/article/pii/S0168583X07008129.

[21] Zaremba S, Kleeven W. Cyclotrons: Magnetic design and beam dynamics. CERN Yellow Rep: School Proc 2017;1:177. https://doi.org/10.23730/CYRSP-2017-001. 177. URL: https://e-publishing.cern.ch/index.php/CYRSP/article/view/99.

[22] van de Walle J, Abs M, Conjat M, Forton E, Henrotin S, Jongen Y, Kleeven W, Mandrillon J, Mandrillon1 P, Verbruggen P. The S2C2: From source to extraction. In: Proceedings of the 21st international conference on cyclotrons and their applications (Cyclotrons'16), Zurich, Switzerland, 11-16 September, 2017. p. 285-9. doi:10.18429/JACoW-Cyclotrons2016-THB01.

[23] Patch SK, Hoff DE, Webb TB, Sobotka LG, Zhao T. Two-stage ionoacoustic range verification leveraging monte carlo and acoustic simulations to stably account for tissue inhomogeneity and accelerator-specific time structure - A simulation study. Med Phys 2018;45(2):783-93. https://doi.org/10.1002/mp.12681.

[24] Cheng C-W, Ferguson SG, Jordan D, Jesseph F, Johnson M, Bouchet L, Mansur D, Wessels B. Impact of the high magnetic field and rf power in a superconducting cyclotron on the operation of a nearby mri facility. J Prot Therapy 2016;2(1):1-10. https://doi.org/10.14319/jpt.21.3. URL: https://wll.com/wp-content/uploads/ Proton-Journal-Article-20-193-1-PB.pdf.

[25] US Department of Health and Human Services: Food and Drug Administration, Mevion s250i, proton radiation beam therapy system premarket notification (510(k)) summary, accessed: 2019-10-18. URL: https://www.accessdata.fda.gov/ cdrh_docs/pdf17/K172848.pdf.

[26] Ebina F, Umezawa M, Nishiuchi H, Aoki T, Hiramoto K, Matsuda K, Umegaki K, Furusaka M. Development of a compact synchrotron for proton beam therapy. Electron Commun Jpn 2017;100(8):34-42. https://doi.org/10.1002/ecj.11972.

[27] Younkin JE, Bues M, Sio TT, Liu W, Ding X, Keole SR, Stoker JB, Shen J. Multiple energy extraction reduces beam delivery time for a synchrotron-based proton spotscanning system. Adv Radiat Oncol 2018;3(3):412-20. https://doi.org/10.1016/j. adro.2018.02.006. URL: http://www.sciencedirect.com/science/article/pii/ S2452109418300319.

[28] Saito K, Nishiuchi H, Totake S, Umezawa M, Nihongi H, Sakurabata H, Moriyama K. Accelerator development for advanced particle beam therapy. In: Proceedings of 11th european particle accelerator conference (EPAC'08), Genoa, Italy, 23-27 June, 2008. p. 1827-29. URL: http://accelconf.web.cern.ch/AccelConf/e08/papers/ tupp129.pdf.

[29] Ungaro D, Degiovanni A, Stabile P, LIGHT: A Linear Accelerator for Proton Therapy. In: Proceedings of north american particle accelerator conference (NAPAC'16), Chicago, IL, USA, October 9-14, 2017. p. 1282-86. doi:10.18429/JACoWNAPAC2016-FRB1IO02

[30] Degiovanni A, Adam J, Murciano DA, Ballestrero S, Morell AB, Bonomi R, et al. Status of the commissioning of the LIGHT prototype. In: Proceedings of the 9th international particle accelerator conference (IPAC'18), Vancouver, BC, Canada, April 29-May 4, 2018. p. 425-8. doi:10.18429/JACoW-IPAC2018-MOPML014.

[31] Chu W, Staples J, Ludewigt B, Renner T, Singh R, Nyman M, et al. Performance specifications for proton medical facility doi:10.2172/10163935. URL: https:// www.osti.gov/biblio/10163935-V460Ns/.

[32] Owen H, MacKay R, Peach K, Smith S. Hadron accelerators for radiotherapy. Contemp Phys 2014;55(2):55-74. https://doi.org/10.1080/00107514.2014. 891313. 
[33] Krämer M, Jäkel O, Haberer T, Kraft G, Schardt D, Weber U. Treatment planning for heavy-ion radiotherapy: physical beam model and dose optimization. Phys Med Biol 2000;45(11):3299-317. https://doi.org/10.1088/0031-9155/45/11/313.

[34] Kraemer M, Trip98 online documentation, accessed: 2019-08-22. URL: http://bio. gsi.de/DOCS/trip98.html.

[35] Bourhis J, Montay-Gruel P, Jorge PG, Bailat C, Petit B, Ollivier J, Jeanneret-Sozzi W, Ozsahin M, Bochud F, Moeckli R, Germond J-F, Vozenin M-C. Clinical translation of FLASH radiotherapy: Why and how? Radiother Oncol 2019;139:11-7. https://doi.org/10.1016/j.radonc.2019.04.008. URL: http://www.sciencedirect. com/science/article/pii/S0167814019303603.

[36] Vozenin M-C, Hendry J, Limoli C. Biological benefits of ultra-high dose rate FLASH radiotherapy: sleeping beauty awoken. Clin Oncol 2019;31(7):407-15. https://doi. org/10.1016/j.clon.2019.04.001. URL: http://www.sciencedirect.com/science/ article/pii/S0936655519301517.

[37] Owen H, Lomax A, Jolly S. Current and future accelerator technologies for charged particle therapy. Nucl Instrum Methods Phys Res Sect A: Accel Spectrom Detect Assoc Equip 2016;809:96-104, advances in detectors and applications for medicine. doi:10.1016/j.nima.2015.08.038. URL: http://www.sciencedirect.com/science/article/pii/S0168900215009729.

[38] Schreuder AN, Shamblin J, Proton therapy delivery: What is needed in the next ten years?. The British Journal of Radiology 2019:20190359. pMID: 31692372. doi:10. 1259/bjr.20190359.

[39] Pedroni E, Meer D, Bula C, Safai S, Zenklusen S. Pencil beam characteristics of the next-generation proton scanning gantry of PSI: design issues and initial commissioning results. Eur Phys J Plus 2011;126(7):66. https://doi.org/10.1140/epjp/ i2011-11066-0.

[40] Schnuerer R, Yap J, Zhang H, Cybulski T, Smith T, Haefeli G, et al. Development of the LHCb VELO detector modules into a standalone. Non-Invas Online Beam Monit Med Acceler Instrum 3(1). doi:10.3390/instruments3010001. URL: https://www. mdpi.com/2410-390X/3/1/1.

[41] Wan W, Brouwer L, Caspi S, Prestemon S, Gerbershagen A, Schippers JM, Robin D. Alternating-gradient canted cosine theta superconducting magnets for future compact proton gantries. Phys Rev ST Accel Beams 2015;18:103501 https://doi.org/10. 1103/PhysRevSTAB.18.103501.

[42] Brouwer L, Caspi S, Hafalia R, Hodgkinson A, Prestemon S, Robin D, Wan W. Design of an achromatic superconducting magnet for a proton therapy gantry. IEEE Trans Appl Supercond 2017;27(4):1-6. https://doi.org/10.1109/TASC.2016.2628305. URL: https://ieeexplore.ieee.org/document/7742387.

[43] Kim J, Yoon M. Design of a compact gantry for carbon-ion beam therapy. Phys Rev Accel Beams 2019;22:101601 https://doi.org/10.1103/PhysRevAccelBeams.22. 101601. URL: https://link.aps.org/doi/10.1103/PhysRevAccelBeams.22.101601.

[44] Keil E, Sessler AM, Trbojevic D. Hadron cancer therapy complex using nonscaling fixed field alternating gradient accelerator and gantry design. Phys Rev ST Accel Beams 2007;10:054701 https://doi.org/10.1103/PhysRevSTAB.10.054701.

[45] Trbojevic D, Parker B, Keil E, Sessler AM. Carbon/proton therapy: A novel gantry design. Phys Rev ST Accel Beams 2007;10:053503 https://doi.org/10.1103/ PhysRevSTAB.10.053503.

[46] Trbojevic D, Morozov V. Innovative superconducting non scaling fixed field alternating gradient isocentric gantry for carbon cancer therapy. In: Proceedings of 2 nd international particle accelerator conference (IPAC11), San Sebastian, Spain, 4-9 September, 2011. p. 2544-6. URL: http://accelconf.web.cern.ch/AccelConf/ IPAC2011/papers/weps029.pdf.

[47] Nesteruk KP, Calzolaio C, Meer D, Rizzoglio V, Seidel M, Schippers JM. Large en ergy acceptance gantry for proton therapy utilizing superconducting technology. Phys Med Biol 2019;64(17):175007 https://doi.org/10.1088/1361-6560/ab2f5f.

[48] Seville A, Adams D, Appelbee C, Bayley D, Farthing N, Gardner I, et al. Progress on dual harmonic acceleration on the isis synchrotron. In: Proceedings of 22nd IEEE particle accelerator conference (PAC), Albuquerque, NM, USA, June 25-29, 2007. p. 1649-1651. doi:10.1109/PAC.2007.4440852.

[49] Adams D. Private communication.

[50] Trbojevic D, Gupta R, Parker B, Keil E, Sessler A. A dramatically reduced size in the gantry design for the proton-carbon therapy. In: Proceedings of 10th european particle accelerator conference (EPAC'06), Edinburgh, Scotland, 26-30 June, 2006. p. 2352-4. URL: http://accelconf.web.cern.ch/AccelConf/e06/PAPERS/ WEPCH180.PDF.

[51] Klimpki G, Psoroulas S, Bula C, Rechsteiner U, Eichin M, Weber DC, Lomax A, Meer $D$. A beam monitoring and validation system for continuous line scanning in proton therapy. Phys Med Biol 2017;62(15):6126-43. https://doi.org/10.1088/13616560/aa772e.

[52] Di Martino F, Giannelli M, Traino AC, Lazzeri M. Ion recombination correction for very high dose-per-pulse high-energy electron beams. Med Phys 2005;32(7Part1):2204-10. https://doi.org/10.1118/1.1940167.

[53] Petersson K, Jaccard M, Germond J-F, Buchillier T, Bochud F, Bourhis J, Vozenin MC, Bailat C. High dose-per-pulse electron beam dosimetry - A model to correct for the ion recombination in the advanced Markus ionization chamber. Med Phys 2017;44(3):1157-67. https://doi.org/10.1002/mp.12111.

[54] Seravalli E, de Boer MR, Geurink F, Huizenga J, Kreuger R, Schippers JM, van Eijk CWE. 2d dosimetry in a proton beam with a scintillating GEM detector. Phys Med Biol 2009;54(12):3755-71. https://doi.org/10.1088/0031-9155/54/12/010.

[55] Simeonov Y, Weber U, Penchev P, Ringbæk TP, Schuy C, Brons S, EngenhartCabillic R, Bliedtner J, Zink K. 3D range-modulator for scanned particle therapy: development, Monte Carlo simulations and experimental evaluation. Phys Med Biol 2017;62(17):7075-96. https://doi.org/10.1088/1361-6560/aa81f4.

[56] Yokokawa K, Furusaka M, Matsuura T, Hirayama S, Umegaki K. A new SOBP-formation method by superposing specially shaped Bragg curves formed by a mini- ridge filter for spot scanning in proton beam therapy. Phys Med 2019;67:70-6. https://doi.org/10.1016/j.ejmp.2019.10.036. URL: http://www.sciencedirect. com/science/article/pii/S1120179719304867.

[57] Tommasino F, Rovituso M, Bortoli E, Tessa CL, Petringa G, Lorentini S, Verroi E, Simeonov Y, Weber U, Cirrone P, Schwarz M, Durante M, Scifoni E. A new facility for proton radiobiology at the Trento proton therapy centre: Design and implementation. Phys Med 2019;58:99-106. https://doi.org/10.1016/j.ejmp.2019. 02.001. URL: http://www.sciencedirect.com/science/article/pii/ S1120179719300213.

[58] IBA, Flash irradiation delivered in a clinical treatment room: successful flash irradiation at isocenter in IBA's proteus solution gantry room, accessed: 2019-10-01. URL: https://iba-worldwide.com/content/pt/proton-flash-irradiation-deliveredclinical-treatment-room.

[59] Flanz JB, et al. Operation of a cyclotron based proton therapy facility. In: Proceedings of the 17th international conference on cyclotrons and their applications (Cyclotrons'04), Tokyo, Japan, 18-22 October, 2004. URL: http://accelconf. web.cern.ch/c04/data/CYC2004_papers/19B1.pdf.

[60] Gerbershagen A, Baumgarten C, Kiselev D, van der Meer R, Risters Y, Schippers M. Measurements and simulations of boron carbide as degrader material for proton therapy. Phys Med Biol 2016;61(14):N337-48. https://doi.org/10.1088/00319155/61/14/n337.

[61] Oponowicz E, Owen H, Psoroulas S, Meer D. Geometry optimisation of graphite energy degrader for proton therapy. Phys Med 2020;76:227-35. https://doi.org/10 1016/j.ejmp.2020.06.023. URL: http://www.sciencedirect.com/science/article/ $\mathrm{pii} / \mathrm{S} 1120179720301575$

[62] Garonna A, Farinon F, Kronberger M, Kulenkampff T, Kurfürst C, Myalski S, et al. Status of proton beam commissioning of the medaustron particle therapy accelerator. In: Proceedings of 7 th international particle accelerator conference (IPAC'16), Busan, Korea, May 8-13, 2016. p. 3176-9. doi:10.18429/JACoWIPAC2016-THOAB01.

[63] Mizushima K, Katagiri K, Iwata Y, Furukawa T, Fujimoto T, Sato S, et al. Experimental studies of systematic multiple-energy operation at HIMAC synchrotron. Nucl Instrum Methods Phys Res Sec B: Beam Interact Mater Atoms 2014;331:243-247, 11th European Conference on Accelerators in Applied Research and Technology. doi:10.1016/j.nimb.2013.12.033. URL: http://www.sciencedirect. com/science/article/pii/S0168583X14001074.

[64] Thomason J. The ISIS spallation neutron and muon source: the first thirty-three years. Nucl Instrum Methods Phys Res Sect A: Accel Spectrom Detect Assoc Equip 2019;917:61-7. https://doi.org/10.1016/j.nima.2018.11.129. URL: http://www. sciencedirect.com/science/article/pii/S0168900218317820.

[65] Pullia MG, Bressi E, Falbo L, Priano C, Rossi S, Viviani C, et al. Betatron core driven slow extraction at CNAO and MedAustron. In: Proceedings of 7th international particle accelerator conference (IPAC'16), Busan, Korea, May 8-13, 2016. p. 1330-3. doi:10.18429/JACoW-IPAC2016-TUPMR037. URL: http://jacow.org/ ipac2016/papers/tupmr037.pdf.

[66] Furukawa T, Noda K, Muramatsu M, Uesugi T, Shibuya S, Kawai H, Takada E, Yamada S. Global spill control in RF-knockout slow-extraction. Nucl Instrum Methods Phys Res Sec A: Accel Spect Detect Assoc Equip 2004;522(3):196-204. https://doi.org/10.1016/j.nima.2003.11.395. URL: http://www.sciencedirect. com/science/article/pii/S016890020303331X.

[67] Schoemers C, Feldmeier E, Naumann J, Panse R, Peters A, Haberer T. The intensity feedback system at heidelberg ion-beam therapy centre. Nucl Instrum Methods Phys Res Sec A: Accel Spect Detect Assoc Equip 2015;795:92-9. https://doi.org/10. 1016/j.nima.2015.05.054. URL: http://www.sciencedirect.com/science/article/ pii/S0168900215007135.

[68] Krantz C, Fischer T, Kröck B, Scheeler U, Weber A, Witt M, et al. Slow extraction techniques at the marburg ion-beam therapy centre. In: Proceedings of 9th international particle accelerator conference (IPAC'18), Vancouver, BC, Canada, April 29-May 4, 2018. p. 1084-6. doi:10.18429/JACoW-IPAC2018-TUPAL036. URL: http://jacow.org/ipac2018/papers/tupal036.pdf.

[69] DeFranco A, Adler L, Farinon F, Gambino N, Guidoboni G, Kowarik G, et al. Slow extraction optimization at the MedAustron ion therapy center: implementation of front end acceleration and RF knock out. In: Proceedings of 9th international particle accelerator conference (IPAC'18), Vancouver, BC, Canada, April 29-May 4, 2018. p. 453-6. doi:10.18429/JACoW-IPAC2018-MOPML025.

[70] IBA, Proteus ONE. Accessed: 2019-08-13. URL: https://iba-worldwide.com/protontherapy/proton-therapy-solutions/proteus-one.

[71] Mevion Medical Systems, Proton therapy products. Accessed: 2019-08-19. URL: https://www.mevion.com/products.

[72] Tesse R, Dubus A, Gnacadja E, Hernalsteens C, Pauly N, Boogert S, et al. Shields, seamless beam and radiation transport simulations of IBA proteus systems using BDSIM. In: Proceedings of the international computational accelerator physics conference (ICAP'18), Key West, FL, USA, 20-24 October, 2018. URL: http://accelconf.web.cern.ch/AccelConf/icap2018/talks/supag11_talk.pdf.

[73] IBA. Flash irradiation delivered in a ProteusONE treatment room: successful ultra high dose rate delivered at isocenter in IBA's compact proton therapy solution. Accessed: 2019-10-04. URL: https://iba-worldwide.com/content/flash-irradiationdelivered-proteus-one-treatment-room.

[74] Amaldi U, Braccini S, Puggioni P. High frequency linacs for hadrontherapy. Rev Accel Sci Technol 2009;02(01):111-31. https://doi.org/10.1142/ S179362680900020X.

[75] Kolano A, Degiovanni A, Farr J, Investigation on flash therapy using a high-frequency linac for protons. In: Particle therapy co-operative group 58 (PTCOG58), Manchester, UK, June 10-June 15, 2019. 